\title{
Efficient Self-Adjusting, Similarity-based Location Fingerprinting with Passive UHF RFID
}

\author{
Philipp Vorst, Artur Koch, and Andreas Zell \\ Computer Science Department \\ University of Tübingen \\ Tübingen, Germany \\ Email: \{philipp.vorst, artur.koch, andreas.zell $\} @$ uni-tuebingen.de
}

\begin{abstract}
In this paper we present extensive experimental results of location fingerprinting with passive UHF radio-frequency identification (RFID). As recent passive RFID hardware provides information about received signal strength (RSS), we evaluate its usefulness in the context of fingerprinting based on classical vector similarity measures. We analyze the impact of decisive parameters of the applied approach and also select them automatically via cross-validation, including the most appropriate similarity measure. A further novelty is an RSS thresholding mechanism which reduces the computational demands of comparing fingerprints. This technique is especially useful in surroundings which are densely equipped with RFID tags, such as future supermarkets or logistic centers. We conducted realworld experiments with a mobile robot and two different RFID readers. Results are reported both for global localization in each time frame and for time-filtered position tracking. We provide the experimental data of this work for download.
\end{abstract}

\section{INTRODUCTION}

The indoor localization of mobile agents has been a vital research topic in recent years. It was identified as a key prerequisite for location-based services and autonomous robots. In the context of radio-frequency identification (RFID), a number of either propagation model-based or fingerprinting techniques have been developed. In this work, we assume the setup of a mobile RFID reader whose position is to be determined. Stationary passive transponders are spread over the environment in arbitrary locations. This setup is particularly relevant in economical applications such as RFID-supported warehousing, logistics, and retail.

We focus on the extension of our recently published location fingerprinting approach [1] using next generation ultra-high frequency (UHF) RFID readers. There, we matched current RFID measurements and reference measurements at known positions efficiently by means of classical similarity measures. Based on this comparison, a position was estimated via particle filtering. In this paper we oppose particle filtering to weighted k-nearest neighbors (WKNN). Moreover, in our previous approach similarities between single fingerprints were solely based upon tag IDs and the respective detection counts during a read operation. The latest generation of readers, however, additionally report the received signal strength (RSS) of the responses of all tags. Therefore, we extend our approach by RSS fingerprints.

By cross-validation we configure central parameters of the approach automatically, e.g., the number of nearest neighbors as well as type and parameterization of the similarity measure. As a result we obtain the best assignment of parameters for the reference data set. This choice will be used in the online localization process to improve the localization errors. Furthermore, we propose a thresholding operation on signal strength that may be used to speed up positioning on systems with less computational power.

The remainder of the paper is structured as follows. In Sect. II we survey related works. Thereafter, we detail our localization approach in Sect. III. Experimental results are provided in Sect. IV. Finally, in Sect. V, we draw conclusions.

\section{RELATED WORK}

This work is related to a variety of radio location fingerprinting approaches. Bahl et al. [2] developed the in-building system RADAR, in which WLAN signal strength measurements were used for combined localization via fingerprinting and signal propagation modeling. Ladd et al. [3] extended RADAR on a robotic platform by Markov localization. Li et al. [4] compared WKNN and Markov localization based on WLAN signal strength measurements. Examples of fingerprinting with other proprietary active RFID systems are LANDMARC [5] and the work by Yamano et al. [6]. Widyawan et al. [7] performed particle filtering and probabilistic 1-nearest neighbor with signal strength in simulation.
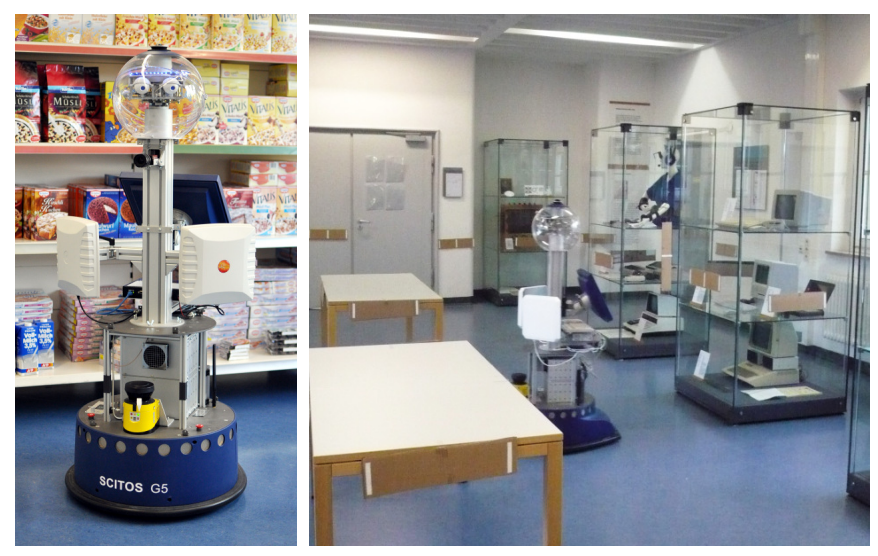

Fig. 1. Photographs of one experimental platform (left, RFID antennas white) and a part of the environment (right) with RFID tags (white, on cardboards) 
Ferris et al. [8] localized by means of particle filtering on mixed graph/free space representations. Signal strength models were learned via Gaussian processes. This approach was later applied by Joho et al. [9] with RSS from passive UHF RFID. Sayrafian et al. [10] applied distance measures to fingerprints of the direction-depending power spectrum of a rotating antenna. Lim and Zhang [11] performed deterministic fingerprinting with passive UHF RFID tags on the ceiling. Schneegans et al. [12] performed fingerprinting with Bayes estimates of detection rates in the global frame of references.

Some approaches employ connectivity rates of active RF nodes, which resembles the detection rates of passive transponders. For instance, Bargh et al. presented location fingerprinting at room-level accuracy, using response rates of Bluetooth dongles [13]. Denby et al. compared Gaussian processes, knearest neighbors, and support vector machines (SVMs) for full-band GSM fingerprinting in a city flat at room-level accuracy [14].

A number of approaches combine particle filtering and RFID with signal strength information. They are typically based on a model of radio propagation between two nodes (e.g., [15]), few others rely on fingerprinting (e.g., [16]).

Wolf et al. [17] developed a method for appearance-based localization. Being a visual fingerprinting approach, it is related to this work, also because the particle filter embedding was similar. Another example is Huhle et al. [18], who applied Gaussian process learning on omnidirectional images to Monte Carlo localization.

Gaussian processes and SVMs typically involve the automatic search for hyperparameters. Some other related fingerprinting approaches using (dis)similarity measures do employ cross-validation for parameter selection (e.g., [19], [20], [21]). To our knowledge, however, understanding the similarity measure itself as an estimation parameter is novel.

\section{Similarity-BASED FINGERPRINTING}

\section{A. Location Fingerprinting}

Location fingerprinting denotes the class of localization methods which determine the position of a mobile system from a set of sensor measurements taken at that location. By comparing current observations to observations recorded at reference locations during a training/calibration stage, the position of the system can be estimated later. An explicit model of the interaction between the sensor and the surroundings is usually not required for that comparison.

Radio location fingerprinting (e.g., [22]) utilizes the identification mechanisms of passive RFID or active radio standards like WLAN, Bluetooth, and GSM. Fingerprints consist of the identifiers of devices in range and information about signal strength, link quality, or detection rates. In the context of wireless communication, location fingerprinting is particularly motivated by the fact that it is difficult to predict radio propagation accurately. Compared to model-based localization, fingerprinting is usually more accurate: The a priori recorded signal distribution in the global frame, including hard to model, location-specific propagation effects, serves as a
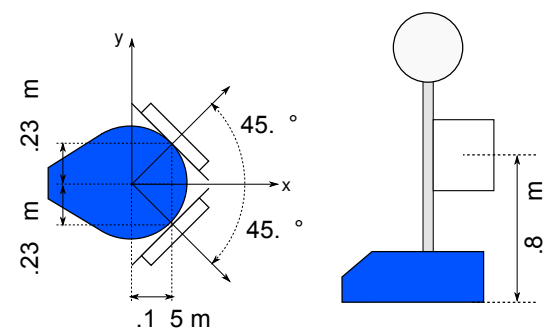

Fig. 2. Top view (left) and side view (right) of the SCITOS robot and the two attached RFID antennas.

reference for localization without explicit modeling. Therefore, we follow the fingerprinting paradigm in this work.

A disadvantage of fingerprinting is that it requires the prior collection of reference measurements. Yet, a number of solutions to this issue have been proposed recently, e.g., [23], [24], [25]. For a comparison and discussion of localization methods, we refer to Seco et al. [26].

Our fingerprinting solution represents RFID measurements as vectors. Similarities between the current observation and the reference measurements in signal space permit to deduce a metric location estimate for the mobile system. For example, the position of the system can either be computed by the similarity-weighted mean of the positions of the $k$ most similar reference fingerprints, or it can be estimated via particle filtering with a likelihood function derived from fingerprint similarities. The former solution has been the classical approach to radio location fingerprinting (cf. [22]). The scenario with passive UHF, however, as investigated also in this paper, is different because:

- The read range of passive RFID is smaller than of WLAN or Bluetooth; at the same time, the density of RFID tags serving as "anchor nodes" can be expected to be two to four orders of magnitude larger if tags are used for pallet, carton, or item labeling.

- RFID readers of the relevant industry standards EPC Class 1 Gen. 2 and ISO/IEC 18000-6C report tag IDs and detection counts, but not necessarily received signal strength (RSS) or angle of arrival. A number of recent RFID readers, however, do provide RSS values. In this paper we aim at comparing the impact of the measured variable on localization accuracy.

- The relative angle to the RFID tag (Fig. 3) and also the difference of orientations of RFID tag and reader antenna reveal major influence on the detectability of the tag. Moreover, we aim at estimating the pose of the mobile platform in this paper, i.e., both position and orientation. The use of two perpendicularly mounted antennas (Fig. 2, left) supports solving this issue, similar to the original setup by Hähnel et al. [27].

\section{B. Representation of Fingerprints}

We represent an RFID measurement at time step $t$ by a tuple $\mathbf{f}_{t}=\left(\mathbf{f}_{t}^{(1)}, \ldots, \mathbf{f}_{t}^{(A)}\right)$ which comprises the readings at the $A$ reader antennas. Let $L$ be the number of RFID 


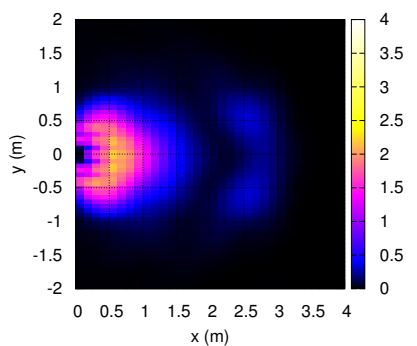

(a)

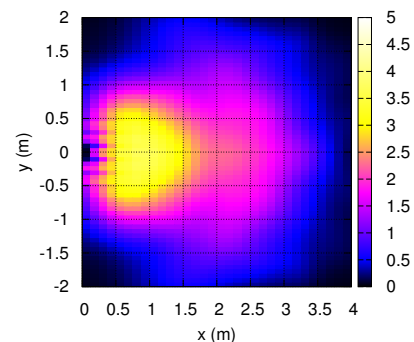

(b)

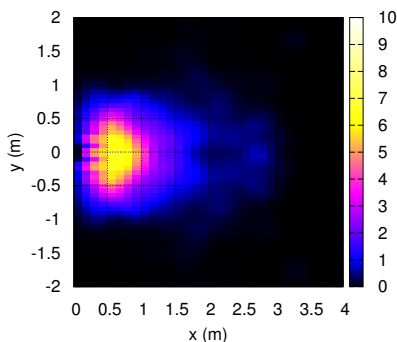

(c)

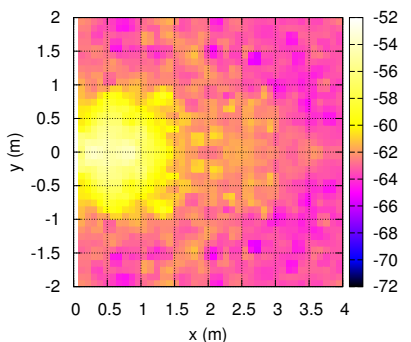

(d)

Fig. 3. Illustrations of the read characteristics (detection rates and received signal strength, RSS) of the employed RFID readers. These models are just for visualization purposes (i.e., not used in this work). (a): Model of smoothed mean detection rates of the Elatec SR-113 reader (30 dBm transmission power) for the detection of a single tag, conditioned on the relative displacement of that tag with respect to a reader antenna. (b): As (a), but for the Impinj Speedway reader. (c): As (b), but at $22.5 \mathrm{dBm}$ transmission power. (d): A model of smoothed RSS (in $\mathrm{dBm}$ ) for the Impinj at $30 \mathrm{dBm}$.

labels in the environment. For each antenna $a$, the vector $\mathbf{f}_{t}^{(a)}=\left(f_{t, 1}^{(a)}, \ldots, f_{t, L}^{(a)}\right)$ contains measured values for all RFID tags. When detection counts are regarded, $f_{t, l}^{(a)} \in \mathbb{N}$ is a nonnegative integer which counts how often tag $l$ was detected by antenna $a$ at time step $t$. If some tag $l$ was not detected, this corresponds to $f_{t, l}^{(a)}=0$. By contrast, when we are interested in RSS values, $f_{t, l}^{(a)} \in \mathbb{R}$ stands for the logarithmic received power, resolved in $\mathrm{dBm}$.

In the training stage, reference measurements are collected. This is often done on a regular mesh of reference positions. Instead, we simply collected measurements during some minutes of explorative traversal of the environment with a mobile robot. The RFID inquiries are annotated with reference positions using laser-based localization [28]. The mapping phase results in a set $\mathbf{m}=\left\{\left(\mathbf{f}_{1}, \mathbf{x}_{1}\right), \ldots,\left(\mathbf{f}_{M}, \mathbf{x}_{M}\right)\right\}$ of RFID reference fingerprints $\mathbf{f}_{i}$ with corresponding poses $\mathbf{x}_{i}=\left(x_{i}, y_{i}, \theta_{i}\right)$. $\left(x_{i}, y_{i}\right)$ are the global Cartesian coordinates of the robot, and $\theta_{i}$ is the global heading of the robot.

Below, we will require vector values to be nonnegative. For this reason, we shift all RSS values by the same constant $\Delta f$ such that the minimum value among the set of reference measurements equals 0 . Missing RSS values can now safely be assumed to be 0 as well. The shift $\Delta f$ will also later be applied to RFID measurements $\mathrm{g}_{t}$ during localization (such that $\left.g_{t, l}^{(a)} \geq 0 \forall l, a, t\right)$.

\section{Similarity-based k-Nearest Neighbors}

In weighted k-nearest neighbors (WKNN) fingerprinting, the position of a mobile device is the weighted mean of those $k$ positions whose reference measurements best match the current observation $\mathrm{g}_{t}$. The weighting and matching is achieved by some similarity measure sim which assesses the pairwise similarity of $\mathbf{g}_{t}$ to each of the $M$ reference fingerprints. Let $\pi$ define a descending sorting of the reference fingerprints by the computed similarity. That is, $\pi:\{1, \ldots, M\} \rightarrow\{1, \ldots, M\}$ such that $\pi(i) \leq \pi(j) \Rightarrow \operatorname{sim}\left(\mathbf{f}_{i}, \mathbf{g}_{t}\right) \geq \operatorname{sim}\left(\mathbf{f}_{j}, \mathbf{g}_{t}\right)$. Then, the WKNN position estimate $\hat{\mathbf{x}}_{t}$ is:

$$
\hat{\mathbf{x}}_{t}=\frac{1}{\sum_{i=1}^{k} \operatorname{sim}\left(\mathbf{f}_{\pi(i)}, \mathbf{g}_{t}\right)} \sum_{i=1}^{k} \mathbf{x}_{\pi(i)} \operatorname{sim}\left(\mathbf{f}_{\pi(i)}, \mathbf{g}_{t}\right)
$$

TABLE I

OVERVIEW OF SIMILARITY MEASURES USED

\begin{tabular}{|l|l|}
\hline $\begin{array}{l}\text { Similarity measure } \\
(+ \text { abbreviation) }\end{array}$ & Formula: $\operatorname{sim}(\mathbf{f}, \mathbf{g})=\ldots$ \\
\hline \hline $\begin{array}{l}\text { Cosine similarity } \\
(\mathrm{COS})\end{array}$ & $\frac{\sum_{l=1}^{L} f_{l} g_{l}}{\sqrt{\sum_{l=1}^{L}\left(f_{l}\right)^{2}} \cdot \sqrt{\sum_{l=1}^{L}\left(g_{l}\right)^{2}}}$ \\
\hline $\begin{array}{l}\text { Histogram intersection } \\
\text { (HI) }\end{array}$ & $\sum_{l=1}^{L} \min \left(f_{l}, g_{l}\right)$ \\
\hline $\begin{array}{l}\text { Bhattacharyya } \\
\text { coefficient (BHA) }\end{array}$ & $\sum_{l=1}^{L} \sqrt{f_{l} g_{l}}$ \\
\hline \hline $\begin{array}{l}\text { Inverse Euclidean } \\
\text { distance (EUCL) }\end{array}$ & $\left(\varepsilon+\sqrt{\sum_{i=1}^{L}\left(f_{i}-g_{i}\right)^{2}}\right)^{-1}$ \\
\hline $\begin{array}{l}\text { Inverse Bray-Curtis } \\
\text { dissimilarity (BC) }\end{array}$ & $\left(\varepsilon+\frac{\sum_{l=1}^{L}\left|f_{l}-g_{l}\right|}{\sum_{l=1}^{L}\left(f_{l}+g_{l}\right)}\right)^{-1}$ \\
\hline
\end{tabular}

If only $k^{\prime}<k$ fingerprints reveal nonzero similarity, one simply proceeds with these $k^{\prime}$ fingerprints.

The similarity function sim must return a nonnegative scalar value which indicates similarity. That is, the disjointness of vectors (each pair of components has at least one value of 0 because the reader detected entirely different tags) results in a similarity of zero. On the other hand, a good match yields a large positive score (the intersection of common tag IDs in both measurements is large). Example similarity functions are cosine similarity and histogram intersection (Table I).

The dual type of functions - dissimilarity measures - can also be used. The Euclidean distance, for instance, has widely been employed for many variants of k-nearest neighbors fingerprinting. Dissimilarities, however, must be transformed to similarities first. For this purpose, we transform a dissimilarity function $d(\cdot, \cdot)$ into a similarity function $\operatorname{sim}(\cdot, \cdot)$ by

$$
\operatorname{sim}\left(\mathbf{f}, \mathbf{g}_{t}\right)=1 /\left(\varepsilon+d\left(\mathbf{f}, \mathbf{g}_{t}\right)\right)
$$

for some constant $\varepsilon>0$. As argued in [1], the parameter $\varepsilon$ should be subject to optimization. One contribution of this work is the automatic choice of $\varepsilon$ via cross-validation. Table I lists all measures employed for this work. We also refer to [1] for details on the functions.

To be precise, similarities $\operatorname{sim}\left(\mathbf{g}_{t}^{(a)}, \mathbf{f}_{i}^{(a)}\right)$ are computed individually for all antennas $a, a=1, \ldots, A$. Then, the similarities at all antennas are integrated to a single scalar 
by averaging, weighted by the number of detected tags at the respective antenna:

$$
\begin{aligned}
\operatorname{sim}\left(\mathbf{g}_{t}, \mathbf{f}_{i}\right) & =\sum_{a=1}^{A} \operatorname{sim}\left(\mathbf{g}_{t}^{(a)}, \mathbf{f}_{i}^{(a)}\right) \cdot \frac{n\left(\mathbf{g}_{t}^{(a)}, \mathbf{f}_{i}^{(a)}\right)}{\sum_{a=1}^{A} n\left(\mathbf{g}_{t}^{(a)}, \mathbf{f}_{i}^{(a)}\right)} \\
\text { where } & n\left(\mathbf{g}_{t}^{(a)}, \mathbf{f}_{i}^{(a)}\right):=\max \left(\left|\mathbf{g}_{t}^{(a)}\right|,\left|\mathbf{f}_{i}^{(a)}\right|\right)
\end{aligned}
$$

be the maximum number of detected tags in $\mathbf{g}_{t}^{(a)}$ and $\mathbf{f}_{i}^{(a)}$ (i.e., $\left.\left|\mathbf{g}_{t}\right|:=\sum_{1 \leq i \leq L: g_{i}>0} 1\right)$.

\section{Cross-Validation for Parameter Adjustment}

The approach so far relies on three decisive parameters which reveal impact on the localization result: The number of considered neighbors, $k$, the distance-to-similarity conversion parameter, $\varepsilon$, and the choice of similarity measure, sim. It is desirable to optimally configure those values automatically for every possible hardware setup and environment. Inspired by recent papers [19], [20], [21], we employ cross-validation (CV: e.g., see [29] for details) for the automatic choice of the best (or close to optimal) parameter set: We split the reference measurements (i.e., the calibration/training data) into $c$ folds (in our case, $c=5$ ). Then, we evaluate the localization performance on each of the $c$ folds, using the other $c-1$ folds as reference data. The test is repeated for different possible combinations of parameter values. The combination with the best localization accuracy on the training data is finally selected for localization.

Cross-validation can be computationally expensive because of combinatorial explosion. Still, it needs to be performed only once, between calibration stage and actual localization. Moreover, the experimental results in Sect. IV indicate that $\mathrm{CV}$ can be performed fast for a reasonably limited parameter subspace.

\section{E. RSS Thresholding}

The run time of computing the similarity between two RFID fingerprints increases linearly with the number of detected tags in the two of them. This value can be large in densely tagged target surroundings such as in warehousing or logistics. Computational time can be saved if the sizes of the measurements get decreased: We propose to replace each RFID measurement $\mathbf{f}$ (either reference or localization measurement) by a more sparse representation $\left.\mathbf{f}\right|_{\geq \vartheta}$, where $\vartheta$ serves as a minimum threshold on RSS values:

$$
\begin{aligned}
\left.\mathbf{f}\right|_{\geq \vartheta}(\mathbf{f}) & :=\left(\tau\left(f_{1}\right), \ldots, \tau\left(f_{L}\right)\right) \\
\text { with } \quad \tau(x) & := \begin{cases}x & x \geq \vartheta \\
0 & \text { else }\end{cases}
\end{aligned}
$$

Equation (5) is well-defined because RSS values were shifted such that the minimum RSS value corresponds to 0 (cf. Sect. III-B).

Thresholding leads to more components with a value of 0 among observation vectors. If the measured values for a specific tag ID $l$ in two compared vectors are both zero, these components will be omitted in the evaluation of the similarity (or dissimilarity) function. This speeds up computation times. In opposition, observations of tags with larger RSS values will not be pruned. They represent non-spurious readings. Larger RSS values embody tags close to the antenna. They are likely to well constrain the current position of the mobile platform. In Sect. IV we will show that thresholding retains sufficient information to still allow for comparable localization accuracy.

\section{F. Particle Filtering and Observation Modeling}

In our earlier paper [1] we showed how to embed k-nearest neighbors fingerprinting into a particle filter. In the work at hand, we apply the same technique and briefly summarize it below for the sake of the self-containedness of this paper.

A particle filter [30] is a probabilistic method to estimate a not directly observable, dynamic state, e.g., the pose of the robot. Being a nonparametric Bayes filter, it recursively approximates a density over robot locations by a discrete set $\left\{\left(\mathbf{x}_{t}^{(i)}, w_{t}^{(i)}\right)\right\}_{1 \leq i \leq N}$ of $N$ samples (particles) for each time step $t$. The $\mathbf{x}_{t}^{(i)}=\left(x_{t}^{(i)}, y_{t}^{(i)}, \theta_{t}^{(i)}\right)$ are pose hypotheses $(2 \mathrm{D}$ position $\left(x_{t}^{(i)}, y_{t}^{(i)}\right)$, orientation $\left.\theta_{t}^{(i)}\right)$ with associated importance weights $w_{t}^{(i)}$. The pose estimate $\hat{\mathbf{x}}_{t}$ at time $t$ is obtained via the weighted mean of sample locations, $\hat{\mathbf{x}}_{t}=\sum_{i=1}^{N} w_{t}^{(i)} \mathbf{x}_{t}^{(i)}$. Particle filtering is known to be robust, accurate, and to permit arbitrarily shaped noise distributions. For details, we refer to [1], [30], [31].

Particle filtering comprises three central, iteratively applied steps: One of them is prediction, in which particles are propagated according to control input (e.g., odometry or inertial data) and a motion model. The second one is the correction step, in which particles are reweighted according to sensor data and an observation model, as detailed below. Resampling, the third type of steps, is applied to select the most likely samples whenever a part of the samples are degenerated.

The RFID-specific model to be adapted is the observation model. It represents the likelihood $p\left(\mathbf{g}_{t} \mid \mathbf{x}_{t}^{(i)}, \mathbf{m}\right)$ of observing the current measurement $\mathbf{g}_{t}$ for a sample placed at position $\mathbf{x}_{t}^{(i)}$, given the $M$ reference fingerprints $\mathbf{m}=$ $\left\{\left(\mathbf{f}_{1}, \mathbf{x}_{1}\right), \ldots,\left(\mathbf{f}_{M}, \mathbf{x}_{M}\right)\right\}$.

We approximated the observation likelihood $p\left(\mathbf{g}_{t} \mid \mathbf{x}, \mathbf{m}\right)$ in pose $\mathbf{x}$ by expanding the map $\mathbf{m}[1]$ :

$$
p\left(\mathbf{g}_{t} \mid \mathbf{x}, \mathbf{m}\right) \approx \nu \sum_{j=1}^{k} \operatorname{sim}\left(\mathbf{g}_{t}, \mathbf{f}_{i_{j}}\right) \exp \left(-\frac{1}{2} d^{2}\left(\mathbf{x}, \mathbf{x}_{i_{j}}\right)\right)
$$

$\nu$ is a normalizer which ensures that the likelihood is a probability density; it does not need to be computed explicitly, because importance weights are normalized after applying the observation model. $d^{2}(\cdot)$ is a kernel which considers both translational and rotational displacement:

$$
d^{2}\left(\mathbf{x}, \mathbf{x}_{i_{j}}\right)=\frac{\left(x-x_{i_{j}}\right)^{2}}{\sigma_{d}^{2}}+\frac{\left(y-y_{i_{j}}\right)^{2}}{\sigma_{d}^{2}}+\frac{\left(\theta \ominus \theta_{i_{j}}\right)^{2}}{\sigma_{r}^{2}}
$$

The $\ominus$ denotes the difference of angles over the interval $[-\pi, \pi] . \sigma_{d}$ and $\sigma_{r}$ are bandwidth parameters for the translational and the rotational distance components, respectively. Visually, the observation model (6) rewards particles with positions and orientations close to similar reference fingerprints. 
TABLE II

DESCRIPTIONS OF EXPERIMENTAL DATA: RANGES (MIN.-MAX.), MEAN VALUES $(\varnothing)$, AND SUMS ( $\Sigma$ ) OF THE FIVE LOG FILES PER DATASET

\begin{tabular}{|c|c|c|c|c|c|c|c|}
\hline Reader & $\begin{array}{l}\text { Transmission } \\
\text { power }(\mathrm{dBm})\end{array}$ & $\begin{array}{c}\text { Number of } \\
\text { RFID measurements }\end{array}$ & $\begin{array}{l}\text { Number of unique tag } \\
\text { IDs detected per log }\end{array}$ & $\begin{array}{l}\text { Trajectory } \\
\text { length }(\mathrm{m})\end{array}$ & $\begin{array}{l}\text { Duration } \\
\text { (s) }\end{array}$ & $\begin{array}{l}\text { Tag detections } \\
\text { per inquiry }\end{array}$ & $\begin{array}{c}\text { Tag count } \\
\text { per detection }\end{array}$ \\
\hline Impinj & 30.0 & $\begin{array}{c}1014-1176 \\
\varnothing: 1064.0, \Sigma: 5320\end{array}$ & $\begin{array}{l}414-452 \\
\varnothing: 429.6\end{array}$ & $\begin{array}{c}206-245 \\
\varnothing: 223.8, \Sigma: 1119\end{array}$ & $\begin{array}{c}528-613 \\
\varnothing: 554.4, \Sigma: 2772\end{array}$ & $\begin{array}{c}0-82 \\
\varnothing: 32.3\end{array}$ & $\begin{array}{c}0-28 \\
\varnothing: 2.9\end{array}$ \\
\hline Impinj & 22.5 & $\begin{array}{c}1011-1147 \\
\varnothing: 1072.2, \Sigma: 5361\end{array}$ & $\begin{array}{l}246-259 \\
\varnothing: 255.4\end{array}$ & $\begin{array}{c}207-239 \\
\varnothing: 229.7, \Sigma: 1148\end{array}$ & $\begin{array}{c}525-597 \\
\varnothing: 557.7, \Sigma: 2788\end{array}$ & $\begin{array}{c}0-31 \\
\varnothing: 7.5\end{array}$ & $\begin{array}{c}0-63 \\
\varnothing: 6.1\end{array}$ \\
\hline Elatec & 30.0 & $\begin{array}{c}1018-1199 \\
\varnothing: 1095.8, \Sigma: 5479\end{array}$ & $\begin{array}{l}249-264 \\
\varnothing: 255.4\end{array}$ & $\begin{array}{c}210-259 \\
\varnothing: 225.9, \Sigma: 1129\end{array}$ & $\begin{array}{c}516-610 \\
\varnothing: 556.7, \Sigma: 2783\end{array}$ & $\begin{array}{c}0-22 \\
\varnothing: 6.7\end{array}$ & $\begin{array}{c}0-26 \\
\varnothing: 3.3\end{array}$ \\
\hline
\end{tabular}

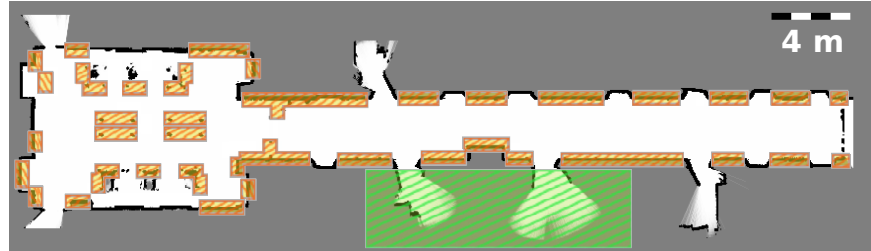

Fig. 4. Grid map of the experimental environment with a traversable area of approx. $160 \mathrm{~m}^{2}$. Areas with RFID tags (attached to walls and furniture) are marked orange and green.

\section{EXPERIMENTAL RESULTS}

We conducted several real-world experiments to validate effectiveness and accuracy of the proposed techniques. Two identical MetraLabs SCITOS G5 robots (Fig. 1) served as experimental platforms. The only difference was the type of on-board, ISO 18000-6C compliant UHF RFID reader: An Elatec SR-113 RFID reader was mounted on the first platform, while the second robot featured an Impinj Speedway reader. The Speedway reader reports detection counts and peak RSS values of all detected tags; the Elatec SR-113 provides detection counts only. The RFID antennas (circularly polarized) were fixed exactly in the same positions as shown in Fig. 2. Characteristic read statistics at a single RFID antenna are visualized in Figs. 3 (a)-(d).

The experimental environment is depicted in Fig. 4. Our setup included approx. 260 RFID tags (type Alien Technology Squiggle, ISO/IEC 18000-6C). The tags were placed on walls and room equipment at different heights between the ground and the level of the RFID antennas. As before [1], the installation was not overly systematic by purpose. Besides that, we tried to spread tags roughly in a balanced distribution along the borders of the corridors. A number of tags in an adjacent laboratory (green area in Fig. 4) were also sometimes accessible by the readers.

We recorded three datasets of five log files each. Each log comprises at least 1000 RFID measurements, odometry, and true positions along one coherent, manually steered trajectory through the environment. RFID measurements were acquired at $2 \mathrm{~Hz}$. Reference positions and ground truth were recorded by means of laser-based Monte Carlo localization as implemented in CARMEN [28]. As shown in Table II, the logs contain the data of approx. 16,000 RFID inquiries from more than $3 \mathrm{~km}$ distance traveled in total. Histograms of RSS and detection counts per tag and inquiry are depicted in Fig. 5.
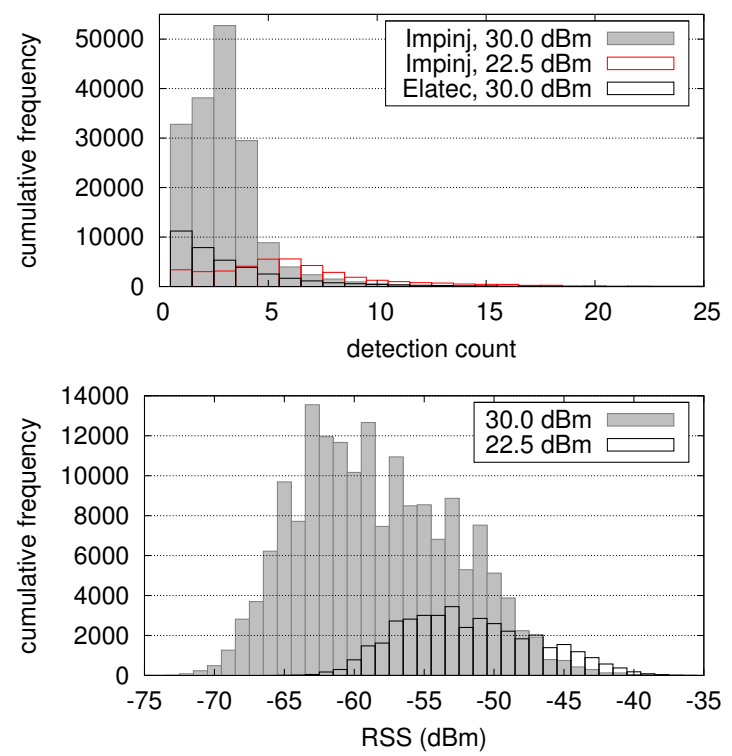

Fig. 5. Distributions of detection rates (top) and RSS values (bottom, Impinj Speedway only) among the different data sets.

The experimental results below reflect the outcome of a large number of trials run on the data in an offline fashion. Each trial stands for localizing the robot on one entire log file, using $M=2000$ fingerprints from two of the remaining $\log$ files as references. So, an outer CV over distinct log files yielded the error values presented in the result figures and tables. The inner cross-validation on reference log files achieved parameter selection.

\section{A. Parameter Cross-Validation}

Tab. III lists the results of our approach including the crossvalidation of parameters and similarity measures. With the Speedway reader and full transmission power, the mean localization errors were approx. $0.4 \mathrm{~m}$ without particle filtering. The mean tracking errors were below $0.25 \mathrm{~m}$ when filtering was applied. With the SR-113, the errors were larger $(0.67 \mathrm{~m}$ and $0.29 \mathrm{~m}$ without and with particle filtering).

Figure 6 (a) illustrates the cross-validation (CV) results (ranging over $k$ and $\varepsilon$ ) for different similarity measures (COS, HIST, BC, EUCL, BHA) and for the case that also the measure itself is selected automatically (AUTO). As a rule, CV selection was superior. Only in case of the SR-113 reader, the $\mathrm{CV}$ result was slightly worse than the optimum, but still comparable. The figure also reveals insight to the ranking of 


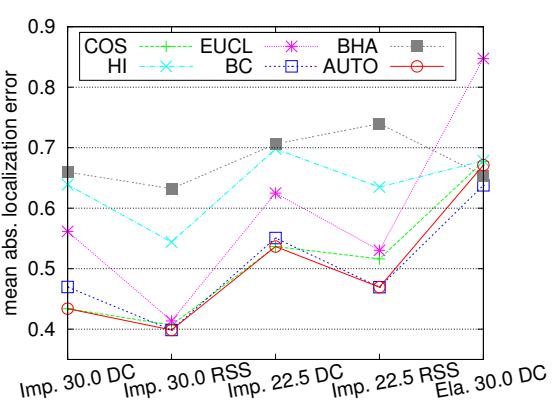

(a)

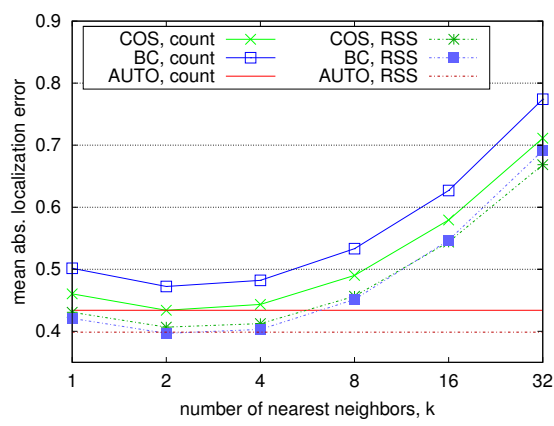

(b)

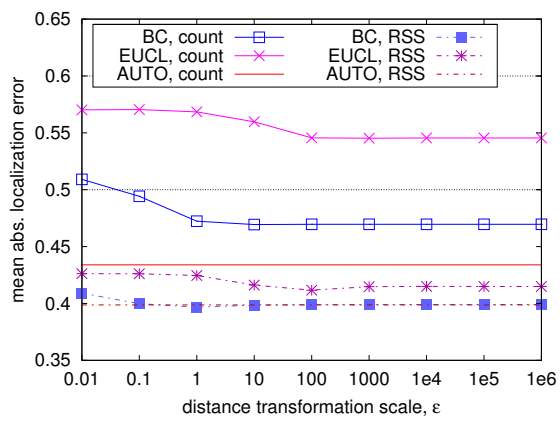

(c)

Fig. 6. Cross-validation results and impacts of the different parameters (without particle filtering): (a) Localization accuracy using cross-validated parameters for different combinations of reader type, transmission power level and measured variable ( $x$-axis). The six curves represent the different similarity and transformed distance measures with varying CV parameter space ( $k$ for similarity measures, $k$ and $\varepsilon$ for distance measures). The red curve (AUTO) represents the mean loc. errors if the CV additionally evaluates similarity/distance measures. (b) Localization accuracy for the cosine and inverse Bray-Curtis similarity under variation of $k$ ( $\varepsilon=1$ fixed). The four curves represent mean loc. errors for combinations of measured variables (RSS/detection count) and the two similarities in use (dataset: Impinj, $30 \mathrm{dBm}$ ). (c) Localization accuracy for the transformed distance measures under variation of $\varepsilon$ when $k=2$ is fixed. The four curves represent mean loc. errors for combinations of measured variables (RSS/detection count) and the two distance measures (dataset: Impinj, $30 \mathrm{dBm}$ ).

the similarity measures. Cosine similarity (COS) and BrayCurtis dissimilarity (BC) performed best.

Figure 6(b) shows that the CV actually selected the best suited number of nearest neighbors, $k$. Moreover, the results of RSS as the measured variable outperform the localization accuracy of detection counts (details follow in Sect. IV-B).

As shown in Fig. 6(c), the best choice of the distance transformation parameter $\varepsilon$ (see (2)) varies with each distance measure. Optimizing $\varepsilon$ yielded improvements of up to $5 \mathrm{~cm}$. A surprising result of Fig. 6(c) is that the localization error gets smaller with larger values of $\varepsilon$. On the other hand, Fig. 7 visualizes that $\varepsilon$ and $k$ mutually affect each other and that higher values of $\varepsilon$ provide less accurate localization results for larger values of $k$. Thus, to achieve optimal precision, $k$ as well as $\varepsilon$ need to be chosen with care.

Although the weights of the fingerprints become very small for large values of $\varepsilon$ (cf. (2) for $\varepsilon \gg d\left(\mathbf{f}, \mathbf{g}_{t}\right)$ ), the order of chosen candidate reference fingerprints is retained. Thus, (1) degrades to a simple mean function, but still yields a satisfactory approximation of the pose due to the similarity in signal space. This characteristic emphasizes the advantage of a particle filter, which uses a prior distribution to focus on likely areas of the location space.

\section{B. RFID Detections vs. RSS}

The influence of the measured variable (RSS vs. detection counts) is depicted in Fig. 6(b) and Tab. III. For all investigated cases, the localization errors were smaller when RSS was employed. The difference is considerable (approx. $7 \mathrm{~cm}$ ) without particle filter and with an RF power of $22.5 \mathrm{dBm}$. Otherwise, RSS improved the positioning accuracy by $1-4 \mathrm{~cm}$ only. The small amount of the deviations may be unexpected, particularly because the range of RSS values is larger than the range of detection counts (cf. Fig. 5). Still, as indicated by Fig. 3(d), the smoothed RSS values differ roughly as much as the smoothed detection counts; they do not appear to distinguish locations better.

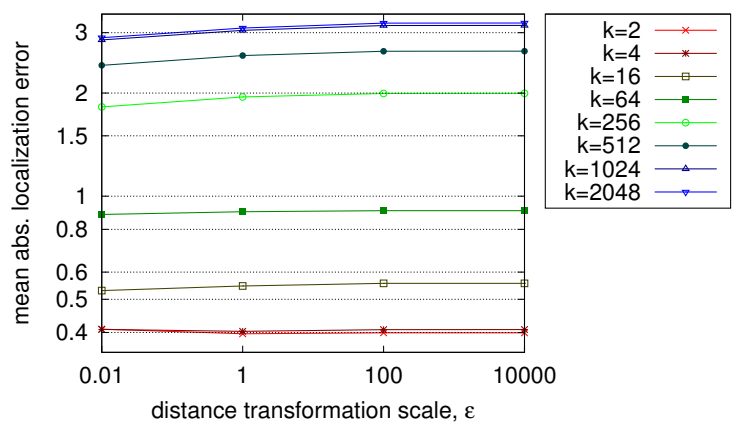

Fig. 7. Mean absolute localization errors for the inverse Bray-Curtis dissimilarity under variation of $k$ and $\varepsilon$.

This finding is similar to the results by Joho et al., who even concluded that a "signal strength model alone was consistently less accurate than the tag detection model" [9].

\section{Impact of Particle Filtering}

Additionally, we analyze the effect of temporal filtering. Before our experiments, the parameters of the motion model had been calibrated using the method by Eliazar and Parr [32]. We applied residual resampling whenever the effective sample size (see. [30]) dropped below $N / 2$. Each experiment was repeated ten times in order to meet the random nature of sampling.

The particle filter results are listed in Tab. III. By contrast to WKNN, we show the accuracy of tracking, i.e., the initial position of the robot was given. This way, the results reflect the long-term accuracy as obtained when the observation model from Sect. III-F is applied, independent of the initial localization error. As can be seen, the mean absolute Cartesian position error is between 0.20 and $0.26 \mathrm{~m}$ for the Impinj Speedway reader, depending on measured variable and transmission power. With the Elatec SR-113, a tracking accuracy of 
TABLE III

LOCALIZATION ERRORS AND INFLUENCE OF PARTICLE FILTERING

USING CROSS-VALIDATION-OPTIMIZED PARAMETERS (STD. DEV. OF MEANS OVER DIFFERENT TRAJECTORIES)

\begin{tabular}{|c|c|c|c|c|c|c|c|c|c|c|}
\hline \multirow{3}{*}{$\begin{array}{l}\text { reader } \\
\text { type }\end{array}$} & \multirow{3}{*}{$\begin{array}{c}\text { measured } \\
\text { variable }\end{array}$} & \multirow{3}{*}{$\begin{array}{l}\text { transm. } \\
\text { power } \\
(\mathrm{dBm})\end{array}$} & \multicolumn{4}{|c|}{ k-nearest neighbors } & \multicolumn{4}{|c|}{ particle filter } \\
\hline & & & \multicolumn{3}{|c|}{ Cartesian error (m) } & \multirow{2}{*}{$\begin{array}{c}\text { rotational error (rad) } \\
\text { mean } \pm \text { std.dev. }\end{array}$} & \multicolumn{3}{|c|}{ Cartesian error (m) } & rotational error (rad) \\
\hline & & & mean \pm std.dev. & $\max$ & median & & mean \pm std.dev. & $\max$ & median & mean \pm std.dev. \\
\hline \multirow{4}{*}{ Impinj } & \multirow{2}{*}{$\begin{array}{c}\text { detection } \\
\text { count }\end{array}$} & 30.0 & $0.433 \pm 0.077$ & 0.624 & 0.435 & $0.204 \pm 0.036$ & $0.216 \pm 0.044$ & 0.346 & 0.213 & $0.066 \pm 0.012$ \\
\hline & & 22.5 & $0.537 \pm 0.079$ & 0.721 & 0.528 & $0.268 \pm 0.054$ & $0.256 \pm 0.046$ & 0.410 & 0.248 & $0.072 \pm 0.010$ \\
\hline & \multirow{2}{*}{ RSS } & 30.0 & $0.399 \pm 0.075$ & 0.588 & 0.412 & $0.201 \pm 0.038$ & $0.203 \pm 0.046$ & 0.333 & 0.198 & $0.065 \pm 0.013$ \\
\hline & & 22.5 & $0.470 \pm 0.081$ & 0.648 & 0.457 & $0.262 \pm 0.055$ & $0.225 \pm 0.044$ & 0.362 & 0.214 & $0.072 \pm 0.010$ \\
\hline Elatec & det. count & 30.0 & $0.671 \pm 0.047$ & 0.778 & 0.679 & $0.325 \pm 0.022$ & $0.286 \pm 0.040$ & 0.406 & 0.281 & $0.079 \pm 0.007$ \\
\hline
\end{tabular}

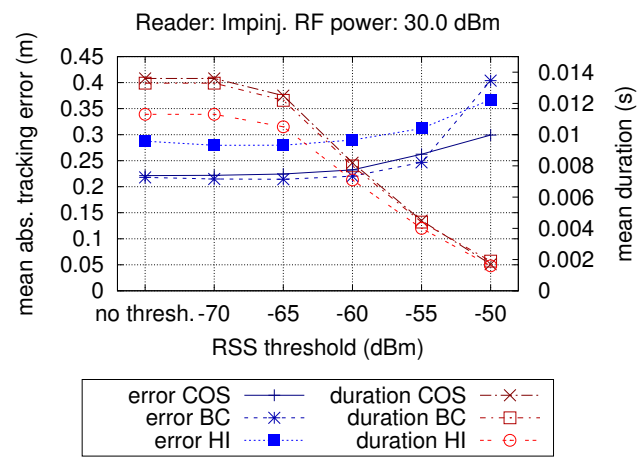

Fig. 8. Timing and accuracy of RSS thresholding: mean position tracking accuracy (blue curves, left vertical axis) vs. computation time per correction step of the particle filter (red curves, right vertical axis) for different thresholds

approx. $0.29 \mathrm{~m}$ was achieved ${ }^{1}$. Furthermore, the mean absolute orientation error was $4.5^{\circ}$ or better for all data when particle filtering was applied. These outcomes underline the benefit of exploiting the temporal correlation of robot positions and the incorporation of odometry.

\section{Impact of RSS Thresholding}

Figure 8 illustrates the results of applying a signal strength threshold as described in Sect. III-E. We used a particle filter with $N=1000$ samples and $M=2000$ reference fingerprints on the Impinj dataset $(30 \mathrm{dBm}$ transmission power). We set $k=4$. The investigated similarity measures were cosine similarity (COS), histogram intersection (HI), and inverse Bray-Curtis dissimilarity (BC). The experiments were run on a Linux PC (dual-core Pentium D $2 \mathrm{GHz}$, only one core used) with 2 GB RAM. Based on the distribution of RSS values in Fig. 5, we tested thresholds between $-70 \mathrm{dBm}$ and $-50 \mathrm{dBm}$.

As can be seen, mean localization errors increased only slightly when only RFID tags with an RSS value below a threshold of less than $-50 \mathrm{dBm}$ were ignored. At the same time, the run time was reduced considerably. For instance, applying a threshold of $-55 \mathrm{dBm}$ decreased the duration of a particle filter correction step from approx. $14 \mathrm{~ms}$ to approx. $5 \mathrm{~ms}$ in case of cosine similarity. At the same time, the mean position

\footnotetext{
${ }^{1}$ In our earlier paper [1], the filter error was better (approx. $0.22 \mathrm{~m}$ ). There, however, the tag density was larger: 8.5 instead of 6.7 tags were detected on average (cf. Tab. II) with a larger mean tag count of 4.1 instead of 3.3 Moreover, for the work at hand, we also recorded measurements in which no tags had been identified. Such inquiries had been ignored in the former data. On the other hand, the CV-enhanced method of the paper at hand revealed approx. $20 \%$ better localization results on the former data [1]
}

tracking error increased from $22 \mathrm{~cm}$ by only $3 \mathrm{~cm}$. However, a threshold of $-50 \mathrm{dBm}$ lead to larger localization errors (mean error of $30 \mathrm{~cm}$ at $2 \mathrm{~ms}$ run time).

\section{E. Impact of Transmission Power}

Table III and Fig. 6 (a) highlight an interesting outcome with respect to the employed transmission power level of the RFID reader: In our experiments, the localization accuracy was better with the greater transmission power. This result may appear surprising. The models in Fig. 3 illustrate a smaller read range when using less power; therefore the certainty about a position should be higher when some transponder is detected. On the other hand, greater transmission power also coincides with a larger number of detections per inquiry and a larger spectrum of RSS values (cf. Fig. 5). These factors seem to outperform the aspect of certainty.

\section{F. Run Times and Memory Consumption}

Figure 8 underlines that our localization approach runs in real time (more than $60 \mathrm{~Hz}$ with $N=1000$ particles) on offthe-shelf PC hardware - even without RSS thresholding. This fact promises that, by adjusting sample size and RSS threshold, our approach is feasible also on less powerful embedded PCs.

Applying a particle filter with 1000 samples consumes approx. 2 ms more per observation than WKNN. The motion model requires less than $1 \mathrm{~ms}$ per step.

During localization, the reference fingerprints were kept in memory without compression. Detection rates and RSS values were represented by double precision. Depending on reader type and transmission power level, between $2 \mathrm{MB}$ (Impinj, $22.5 \mathrm{dBm}$ ) and $13 \mathrm{MB}$ (Impinj, $30.0 \mathrm{dBm}$ ) RAM were required.

The mean duration of the cross-validation was $90.5 \pm 41.5 \mathrm{~s}$ on the same Pentium D $2 \mathrm{GHz}$ PC, but now using both cores.

\section{CONCLUSiON}

In this work we presented extensive experimental results of localizing a robot with an on-board passive UHF RFID reader. We applied fingerprinting with different similarity measures to both detection counts and received signal strength as measured variables. Position and orientation of the mobile system were estimated either by weighted k-nearest neighbors or by additional particle filtering.

Comprehensive experiments showed that we could localize the mobile system with the newer RFID reader at mean Cartesian errors of approx. $0.4 \mathrm{~m}$ without particle filtering. 
With filtering enabled, we tracked the robot in real time at errors slightly larger than $0.2 \mathrm{~m}$. The mean orientation errors obtained were $11.5^{\circ}$ and $4^{\circ}$, respectively. RSS-based localization was slightly superior to detection rate-based localization. Cross-validation, the only preprocessing step required, was shown to be an effective tool to determine essential estimation parameters automatically. The overhead in computational and in programming complexity is negligible. Moreover, we proposed RSS thresholding as a means of reducing computational load in RSS-based localization at the expense of moderately increased errors. We were able to save $70 \%$ run time, while the mean tracking error increased by only $3-4 \mathrm{~cm}$.

Future work may comprise the design of a novel similarity function itself, since it reveals major impact on localization accuracy. Moreover, the fingerprinting community has come up with a variety of fingerprint selection and preprocessing techniques that could be added to our estimation pipeline.

The experimental data of this work can be downloaded from http://www.cogsys.cs.uni-tuebingen.de/datasets/rfid-ta2011. To our knowledge, this is the first publicly available dataset of a passive UHF fingerprinting setup.

\section{ACKNOWLEDGMENT}

This work was funded by the Baden-Württemberg Stiftung in the scope of AmbiSense/BW-FIT.

\section{REFERENCES}

[1] P. Vorst and A. Zell, "A comparison of similarity measures for localization with passive RFID fingerprints," in ISR/ROBOTIK 2010 (Proc. Joint Conf. of ISR 2010 (41st Int. Symp. on Robotics) and ROBOTIK 2010 (6th German Conf. on Robotics)). VDE Verlag, 2010, pp. 354-361.

[2] P. Bahl and V. N. Padmanabhan, "RADAR: An in-building RF-based user location and tracking system," in Proc. 19th Annual Joint Conf. of the IEEE Computer and Communications Societies (INFOCOM), vol. 2. Tel Aviv, Israel: IEEE, Mar. 2000, pp. 775-784.

[3] A. Ladd, K. Bekris, G. Marceau, A. Rudys, L. Kavraki, and D. Wallach, "Robotics-based location sensing using wireless ethernet," Department of Computer Science, Rice University, Tech. Rep. TR02-393, 2002.

[4] B. Li, J. Salter, A. G. Dempster, and C. Rizos, "Indoor positioning techniques based on wireless LAN," in 1st IEEE Int. Conf. on Wireless Broadband and Ultra Wideband Communications, Sydney, Australia, Mar. 2006, paper 113, CD-ROM proceedings.

[5] L. M. Ni, Y. Liu, Y. C. Lau, and A. P. Patil, "LANDMARC: Indoor location sensing using active RFID," ACM Wireless Networks (WINET), vol. 10, no. 6, pp. 701-710, Nov. 2004.

[6] K. Yamano, K. Tanaka, M. Hirayama, E. Kondo, Y. Kimuro, and M. Matsumoto, "Self-localization of mobile robots with RFID system by using support vector machine," in Proc. IEEE/RSJ Int. Conf. on Intelligent Robots and Systems (IROS), vol. 4, 2004, pp. 3756-3761.

[7] Widyawan, M. Klepal, and S. Beauregard, "A novel backtracking particle filter for pattern matching indoor localization," in Proc. First ACM Int. Workshop on Mobile Entity Localization and Tracking in GPS-less Environments (MELT). San Francisco, USA: ACM, 2008, pp. 79-84.

[8] B. Ferris, D. Hähnel, and D. Fox, "Gaussian processes for signal strength-based location estimation," in Robotics: Science and Systems II. Philadelphia, PA, USA: The MIT Press, Aug. 2006.

[9] D. Joho, C. Plagemann, and W. Burgard, "Modeling RFID signal strength and tag detection for localization and mapping," in Proc. IEEE Int. Conf. on Robotics and Automation (ICRA), Kobe, Japan, May 2009, pp. 3160-3165.

[10] K. Sayrafian-Pour and D. Kaspar, "Indoor positioning using spatial power spectrum," in IEEE 16th Int. Symp. on Personal, Indoor and Mobile Radio Communications (PIMRC), vol. 4, 2005, pp. 2722-2726.

[11] A. Lim and K. Zhang, "A robust RFID-based method for precise indoor positioning," in Advances in Applied Artificial Intelligence, ser. LNCS, vol. 4031. Springer, Jun. 2006, pp. 1189-1199.
[12] S. Schneegans, P. Vorst, and A. Zell, "Using RFID snapshots for mobile robot self-localization," in Proc. 3rd European Conf. on Mobile Robots (ECMR), Freiburg, Germany, Sep. 2007, pp. 241-246.

[13] M. S. Bargh and R. de Groote, "Indoor localization based on response rate of Bluetooth inquiries," in Proc. ACM Int. Workshop on Mobile Entity Localization and Tracking in GPS-less Environments (MELT). San Francisco, CA, USA: ACM, Sep. 2008, pp. 49-54.

[14] B. Denby, Y. Oussar, I. Ahriz, and G. Dreyfus, "High-performance indoor localization with full-band GSM fingerprints," in Int. Workshop on Synergies in Communications and Localization (SyCoLo). Dresden, Germany: IEEE, Jun. 2009, pp. 1-5.

[15] A. Raghavan, H. Ananthapadmanaban, M. Sivamurugan, and B. Ravindran, "Accurate mobile robot localization in indoor environments using Bluetooth," in Proc. IEEE Int. Conf. on Robotics and Automation (ICRA). Anchorage, AK, USA: IEEE, May 2010, pp. 4391-4396.

[16] Widyawan, M. Klepal, and D. Pesch, "A Bayesian approach for RFbased indoor localisation," in 4th IEEE Int. Symp. on Wireless Communication Systems (ISWCS), Trondheim, Norway, Oct. 2007.

[17] J. Wolf, W. Burgard, and H. Burkhardt, "Robust vision-based localization by combining an image retrieval system with Monte Carlo localization," IEEE Trans. Robot., vol. 21, no. 2, pp. 208-216, 2005.

[18] B. Huhle, T. Schairer, A. Schilling, and W. Straßer, "Learning to localize using Gaussian process regression on omnidirectional image data," in IEEE/RSJ Int. Conf. on Intelligent Robots and Systems (IROS). IEEE, Oct. 2010, pp. 5208-5213.

[19] D. Felix, E. Hyun, M. McGuire, and M. Sima, "Denoising by spatial domain averaging for wireless local area network terminal localization," in World Academy of Science, Engineering and Technology (WASET), no. 70 , Oct. 2010 , pp. $48-55$.

[20] Y. Han, E. Stuntebeck, J. Stasko, and G. Abowd, "A visual analytics system for radio frequency fingerprinting-based localization," in IEEE Symp. on Visual Analytics Science and Technology (VAST), Atlantic City, NJ, USA, Oct. 2009, pp. 35-42.

[21] G. Jekabsons and V. Zuravlyov, "Refining WI-FI based indoor positioning," in Proc. Int. Scientific Conf. Applied Information and Communication Technologies, Jelgava, Latvia, Apr. 2010, pp. 87-95.

[22] M. B. Kjærgaard, "Indoor positioning with radio location fingerprinting," Ph.D. dissertation, Department of Computer Science, University of Aarhus, Denmark, 2010.

[23] P. Bolliger, "Redpin - adaptive, zero-configuration indoor localization through user collaboration," in Proc. ACM Int. Workshop on Mobile Entity Localization and Tracking in GPS-less Environments (MELT). San Francisco, CA, USA: ACM, Sep. 2008, pp. 55-60.

[24] B. Ferris, D. Fox, and N. Lawrence, "WiFi-SLAM using Gaussian process latent variable models," in Proc. 20th Int. Joint Conf. on Artificial Intelligence (IJCAI), Hyderabad, India, 2007, pp. 2480-2485.

[25] P. Vorst and A. Zell, "Fully autonomous trajectory estimation with long-range passive RFID," in 2010 IEEE Int. Conf. on Robotics and Automation (ICRA), Anchorage, AK, USA, May 2010, pp. 1867-1872.

[26] F. Seco, A. R. Jiménez, C. Prieto, J. Roa, and K. Koutsou, "A survey of mathematical methods for indoor localization," in 6th IEEE Int. Symp. on Intelligent Signal Processing (WISP), Aug. 2009, pp. 9-14.

[27] D. Hähnel, W. Burgard, D. Fox, K. P. Fishkin, and M. Philipose, "Mapping and localization with RFID technology," in Proc. 2004 IEEE Int. Conf. on Robotics and Automation (ICRA). New Orleans, LA, USA: IEEE, April/May 2004, pp. 1015-1020.

[28] M. Montemerlo, N. Roy, and S. Thrun, "Perspectives on standardization in mobile robot programming: The Carnegie Mellon Navigation (CARMEN) Toolkit," in Proc. IEEE/RSJ Int. Conf. on Intelligent Robots and Systems (IROS), vol. 3, Las Vegas, NV, USA, Oct. 2003, pp. 2436-2441.

[29] T. Hastie, R. Tibshirani, and J. Friedman, The Elements of Statistical Learning: Data Mining, Inference, and Prediction, 2nd ed., ser. Springer Series in Statistics. Springer, Feb. 2008.

[30] M. S. Arulampalam, S. Maskell, N. Gordon, and T. Clapp, "A tutorial on particle filters for on-line nonlinear/non-Gaussian Bayesian tracking," IEEE Trans. Signal Process., vol. 50, no. 2, pp. 174-188, February 2002.

[31] I. M. Rekleitis, "A particle filter tutorial for mobile robot localization," Centre for Intelligent Machines, McGill University, Montreal, Québec, Canada, Tech. Rep. TR-CIM-04-02, 2004.

[32] A. I. Eliazar and R. Parr, "Learning probabilistic motion models for mobile robots," in Proc. 21st Int. Conf. on Machine Learning (ICML), Banff, AB, Canada, ser. AICPS, vol. 69. ACM, Jul. 2004, pp. 32-39. 